Psicologia clinica del dolore 
Enrico Molinari • Gianluca Castelnuovo (a cura di)

\section{Psicologia clinica del dolore}

第 Springer 
a cura di

\section{Enrico Molinari}

Professore Ordinario di Psicologia Clinica

Laboratorio di Ricerche Psicologiche

IRCCS Istituto Auxologico Italiano

Facoltà di Psicologia

Università Cattolica del Sacro Cuore

Milano

\section{Gianluca Castelnuovo}

Ricercatore di Psicologia Clinica

Laboratorio di Ricerche Psicologiche

IRCCS Istituto Auxologico Italiano

Facoltà di Psicologia

Università Cattolica del Sacro Cuore

Milano

ISBN 978-88-470-1468-8

e-ISBN 978-88-470-1469-5

DOI 10.1007/978-88-470-1469-5

(C) Springer-Verlag Italia 2010

Quest'opera è protetta dalla legge sul diritto d'autore, e la sua riproduzione è ammessa solo ed esclusivamente nei limiti stabiliti dalla stessa. Le fotocopie per uso personale possono essere effettuate nei limiti del $15 \%$ di ciascun volume dietro pagamento alla SIAE del compenso previsto dall'art. 68 , commi 4 e 5, della legge 22 aprile $1941 \mathrm{n}$. 633. Le riproduzioni per uso non personale e/o oltre il limite del $15 \%$ potranno avvenire solo a seguito di specifica autorizzazione rilasciata da AIDRO, Corso di Porta Romana n. 108, Milano 20122, e-mail segreteria@aidro.org e sito web www.aidro.org.

Tutti i diritti, in particolare quelli relativi alla traduzione, alla ristampa, all'utilizzo di illustrazioni e tabelle, alla citazione orale, alla trasmissione radiofonica o televisiva, alla registrazione su microfilm o in database, o alla riproduzione in qualsiasi altra forma (stampata o elettronica) rimangono riservati anche nel caso di utilizzo parziale. La violazione delle norme comporta le sanzioni previste dalla legge.

L'utilizzo in questa pubblicazione di denominazioni generiche, nomi commerciali, marchi registrati, ecc. anche se non specificatamente identificati, non implica che tali denominazioni o marchi non siano protetti dalle relative leggi e regolamenti.

Responsabilità legale per i prodotti: l'editore non può garantire l'esattezza delle indicazioni sui dosaggi e l'impiego dei prodotti menzionati nella presente opera. Il lettore dovrà di volta in volta verificarne l'esattezza consultando la bibliografia di pertinenza.

\section{$9 \begin{array}{llllllllll}9 & 7 & 6 & 5 & 4 & 3 & 2 & 1\end{array}$}

In copertina: "Luci e colori di Pellicani in volo" di Angelo Antonio Falmi, Firenze, 2009

(www.angeloantoniofalmi.it)

Layout copertina: Simona Colombo, Milano

Impaginazione: Graphostudio, Milano

Stampa: Grafiche Porpora, Segrate (MI)

Stampato in Italia

Springer-Verlag Italia S.r.1., Via Decembrio 28, I-20137 Milano

Springer fa parte di Springer Science+Business Media (www.springer.com) 


\section{Prefazione}

Negli ultimi anni, all'interno del vasto panorama della psicologia clinica e delle sue applicazioni, è emersa sempre più la necessità di studiare il dolore cronico e le sue componenti per poter meglio offrire supporto sia agli psicologi, nel loro operare con le sofferenze dei pazienti, sia alle altre figure sanitarie che possono essere interessate alla conoscenza di aspetti psicologici del dolore per migliorare le proprie attività assistenziali.

Nel testo viene data una specifica attenzione al dolore cronico e alla dimensione trasversale della sofferenza che investe molte problematiche relative al lavoro dei medici, degli psicologi, degli psicoterapeuti, degli infermieri, dei fisioterapisti e di altri operatori sanitari.

Nella prima parte del volume abbiamo voluto offrire una panoramica sul dolore a livello di definizione, classificazione e inquadramento psico-fisiologico.

Nella seconda parte vi è una rassegna sulle principali modalità di valutazione, misurazione e trattamento del dolore stesso con particolare riferimento all'uso dell'ipnosi.

Dopo un "interludio" di riflessione teologica sulla dimensione spirituale del dolore, il libro, nella terza parte, presenta esperienze cliniche e di ricerca condotte presso centri ospedalieri e clinici che hanno ottenuto interessanti risultati in campo scientifico e applicativo. Vi è spazio per l'Istituto Auxologico Italiano, con una ricerca realizzata tramite l'impiego di valutazioni psicofisiologiche presso l'Ospedale San Giuseppe di Piancavallo, e per l'Ambulatorio di Terapia del dolore e il Servizio interno di Psicologia Clinica dell'Istituto Ortopedico Galeazzi di Milano, con il progetto VIRNOPA (VIRtual reality and hypNOsis for the treatment of PAin) condotto in collaborazione con Laboratorio di Psicologia Clinica dell'Università Cattolica di Milano.

Vi è poi il contributo offerto dai medici di famiglia, punto di riferimento sul territorio per i pazienti con dolore cronico; segue la presentazione di una ricerca, ancora in itinere, ma con risultati preliminari promettenti, su possibili utilizzi di tecnologie estreme come la Realtà Virtuale 4D e il lettino vibroacustico nell'integrazione del trattamento del dolore. 
Il libro riporta inoltre un contributo del Dipartimento di Psicologia Clinica della Facoltà di Medicina e Chirurgia dell'Università di Bologna, una riflessione di FederDolore, Coordinamento Italiano dei Centri di Terapia del dolore e un'indagine dell'Onlus NOPAIN, Associazione Italiana per la cura della malattia dolore, sulla situazione dei Servizi esistenti nel territorio italiano relativamente al trattamento del dolore.

Crediamo che per l'intervento sul dolore cronico sia necessaria la collaborazione e l'integrazione di diversi specialisti. In questo volume, attraverso i molteplici contributi di Associazioni, centri di ricerca, medici, psicologi, abbiamo voluto offrire a tutti gli attori coinvolti nella cura uno spazio di confronto teorico e operativo.

Claudio Cricelli, Presidente della SIMG (Società Italiana di Medicina Generale), in un intervento sul tema Combattiamo il dolore per una società più civile, scrive: "Questa assistenza, in accordo con i modelli avanzati di cura nei paesi europei più evoluti, dovrebbe essere erogata da un' 'équipe di curanti' in cui, oltre al medico di famiglia e all'infermiere del territorio, si aggiunge il medico esperto in cure palliative, l'assistente sociale, lo psicologo, l'assistente spirituale, ecc." (Quaderni de Il Sole 24 Ore Sanità, 30-6-2009). Sulla scia di quanto affermato da Cricelli, il volume che presentiamo vuole fornire all' 'équipe di curanti' possibili chiavi di lettura, teorica e pratica, del fenomeno "dolore" e delle sue possibilità di cura.

Milano, gennaio 2010

Enrico Molinari

Gianluca Castelnuovo 


\section{Indice}

Introduzione

Mauro Ercolani

Parte I Conoscere il dolore

1 Il concetto di dolore: definizioni, contesti di interesse e modelli teorici . . . 5

Stefano Cugno, Valentina Forni, Daniele Rovaris, Paola Cuzziol,

Enrico Molinari, Gianluca Castelnuovo

$1.1 \quad$ Le definizioni del dolore $\ldots \ldots \ldots \ldots \ldots \ldots \ldots \ldots \ldots \ldots \ldots$

$1.2 \quad$ I principali contesti di interesse del dolore $\ldots \ldots \ldots \ldots \ldots \ldots \ldots$

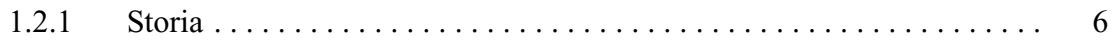

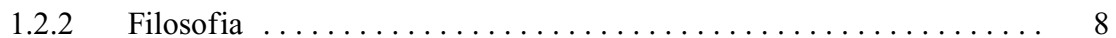

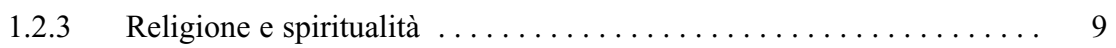

$1.3 \quad$ I principali modelli teorici sul dolore $\ldots \ldots \ldots \ldots \ldots \ldots \ldots \ldots \ldots . \ldots \ldots$

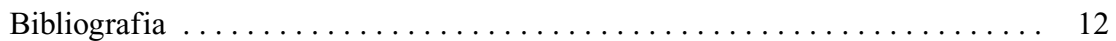

2 I sistemi di classificazione del dolore $\ldots \ldots \ldots \ldots \ldots \ldots \ldots \ldots \ldots$

Stefano Cugno, Valentina Forni, Daniele Rovaris, Paola Cuzziol,

Enrico Molinari, Gianluca Castelnuovo

$2.1 \quad$ I sistemi di classificazione del dolore $\ldots \ldots \ldots \ldots \ldots \ldots \ldots \ldots \ldots$

2.2 I sistemi di classificazione monodimensionale del dolore . . . . . . . . 16

2.2.1 Classificazione basata sull'origine del dolore $\ldots \ldots \ldots \ldots \ldots \ldots . \ldots$

2.2.2 Classificazione basata sull'intensità del dolore . . . . . . . . . . 17

2.2.3 Classificazione basata sul timing del dolore . . . . . . . . . . . 17

2.2.4 Classificazione basata sulla durata del dolore . . . . . . . . . . . . . 17

$2.3 \quad$ I sistemi di classificazione multidimensionale del dolore . . . . . . . 19

$2.4 \quad$ I sistemi di classificazione del dolore in psichiatria $\ldots \ldots \ldots \ldots \ldots 20$

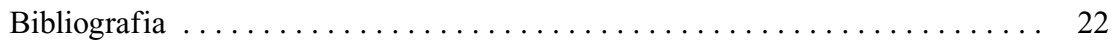




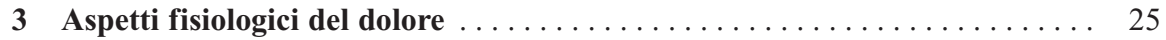

Daniele Rovaris, Stefano Cugno, Valentina Forni, Paola Cuzziol,

Enrico Molinari, Gianluca Castelnuovo

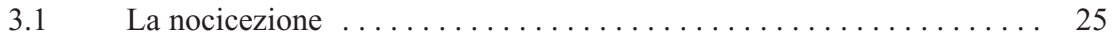

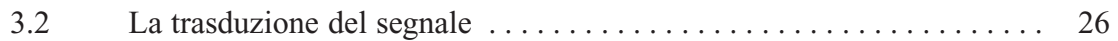

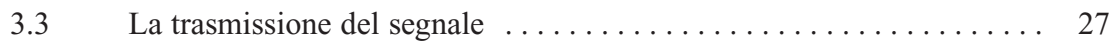

$3.4 \quad$ La modulazione del segnale $\ldots \ldots \ldots \ldots \ldots \ldots \ldots \ldots \ldots \ldots \ldots . \ldots \ldots$

3.4.1 Prima stazione: il midollo spinale $\ldots \ldots \ldots \ldots \ldots \ldots \ldots \ldots \ldots \ldots$

3.4.2 Le vie ascendenti e discendenti del dolore . . . . . . . . . . . 28

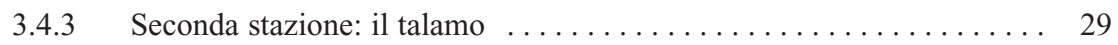

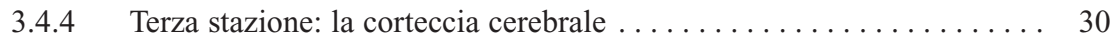

3.5 Teoria del cancello e meccanismi fisiologici modulatori

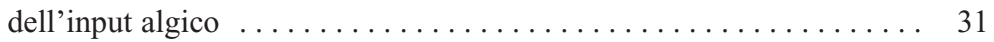

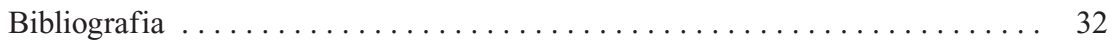

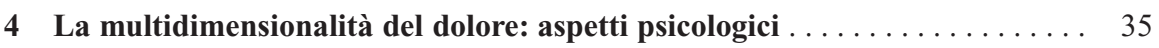

Valentina Forni, Stefano Cugno, Daniele Rovaris, Paola Cuzziol, Enrico Molinari, Gianluca Castelnuovo

4.1 La quotidianità di chi convive con il dolore cronico $\ldots \ldots \ldots \ldots \ldots 36$

$4.2 \quad$ La psicologia del dolore cronico $\ldots \ldots \ldots \ldots \ldots \ldots \ldots \ldots \ldots \ldots$

4.2.1 La componente affettiva del dolore ................. 39

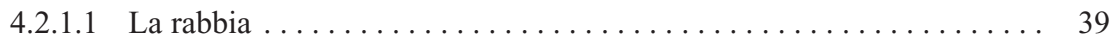

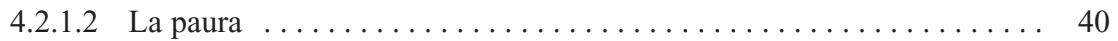

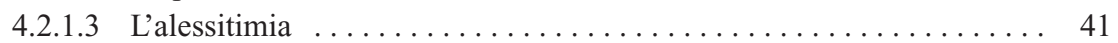

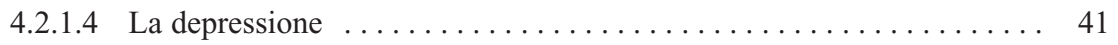

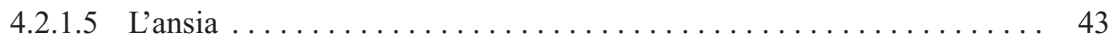

4.2.2 La componente cognitiva del dolore ................. 44

4.2.3 La componente comportamentale del dolore .............. 45

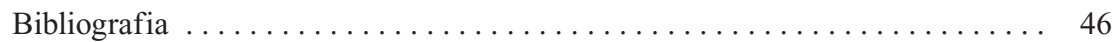

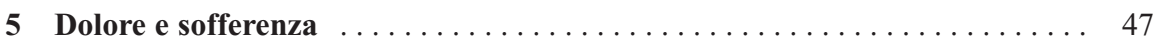

Vincenzo Montrone

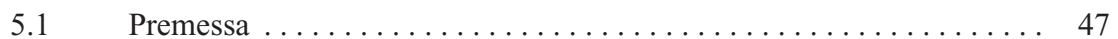

$5.2 \quad$ Il dolore nella cultura delle varie epoche storiche $\ldots \ldots \ldots \ldots \ldots . \ldots 4$

$5.3 \quad$ Il dolore e la sofferenza nella cultura contemporanea $\ldots \ldots \ldots \ldots .51$

Bibliografia ................................. 54 
Parte II Valutare, misurare e curare il dolore $\ldots \ldots \ldots \ldots \ldots \ldots \ldots \ldots \ldots$

$6 \quad$ La valutazione e la misurazione del dolore $\ldots \ldots \ldots \ldots \ldots$. . . . . . . . . . . . 57

Daniele Rovaris, Stefano Cugno, Valentina Forni, Paola Cuzziol, Enrico Molinari, Gianluca Castelnuovo

$6.1 \quad$ Misurazione e valutazione del dolore . . . . . . . . . . . . . . . . . 57

$6.2 \quad$ L'anamnesi e l'esame clinico $\ldots \ldots \ldots \ldots \ldots \ldots \ldots \ldots \ldots \ldots \ldots \ldots$

6.3 Strumenti per la misura dell'intensità/quantità del dolore . . . . . . . 59

6.4 Questionari clinici per la misurazione della qualità del dolore ...... 61

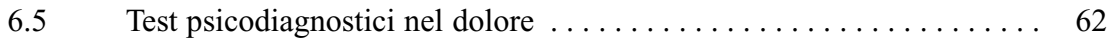

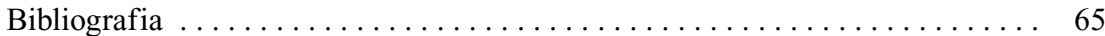

7 Gli approcci terapeutici al trattamento del dolore cronico . . . . . . . . . 67

Gianluca Castelnuovo, Mauro Ercolani, Mario Tavola, Daniele Rovaris, Lara Bellardita, Paola Cuzziol, Enrico Molinari

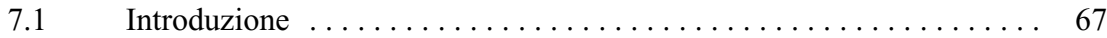

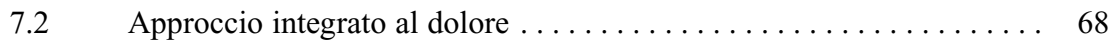

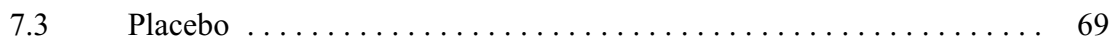

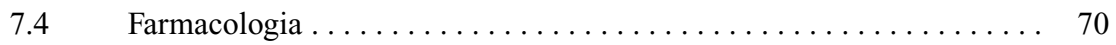

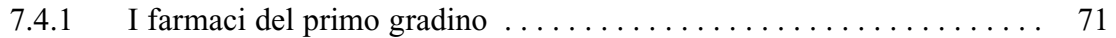

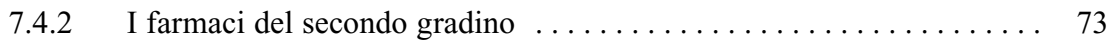

7.4.3 I farmaci del terzo gradino: gli oppioidi forti $\ldots \ldots \ldots \ldots \ldots \ldots 75$

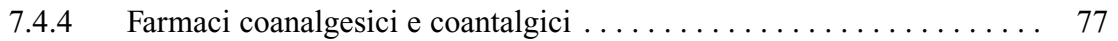

$7.5 \quad$ Trattamenti invasivi $\ldots \ldots \ldots \ldots \ldots \ldots \ldots \ldots \ldots \ldots \ldots \ldots \ldots \ldots$

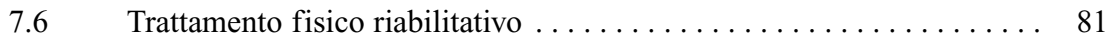

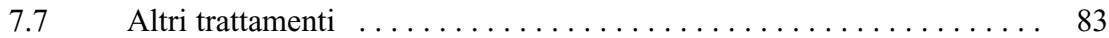

7.8 Trattamenti psicologici e psicoterapie del dolore . . . . . . . . . 85

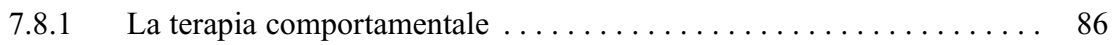

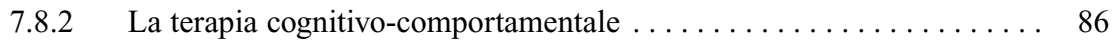

7.8.3 L'approccio psicodinamico: la terapia psicoanalitica . . . . . . . . . . 87

$7.8 .4 \quad$ La terapia di gruppo $\ldots \ldots \ldots \ldots \ldots \ldots \ldots \ldots \ldots \ldots \ldots \ldots$

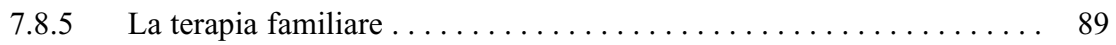

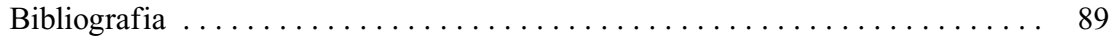

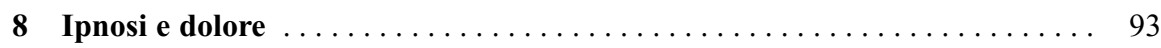

Lara Bellardita, Enrico Molinari, Gianluca Castelnuovo

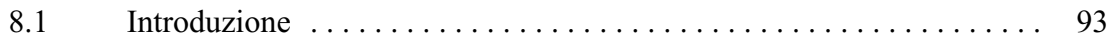

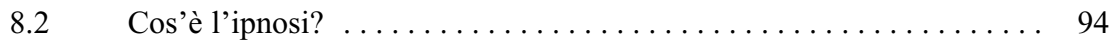

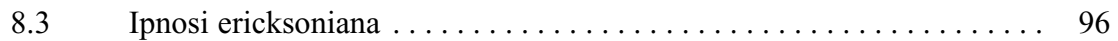

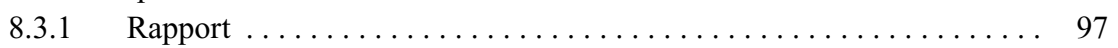

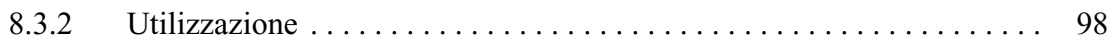

$8.3 .3 \quad$ Suggestione indiretta $\ldots \ldots \ldots \ldots \ldots \ldots \ldots \ldots \ldots \ldots \ldots \ldots$ 
$8.4 \quad$ Utilizzo dell'ipnosi nel trattamento del dolore . . . . . . . . . . . . 99

8.5 Ipnosi e placebo: diversi correlati neurofisiologici . . . . . . . . . 102

8.6 La complessità degli interventi di ipnosi neoericksoniana . . . . . . 103

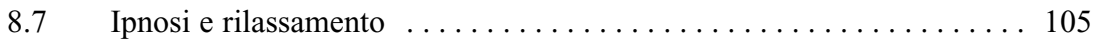

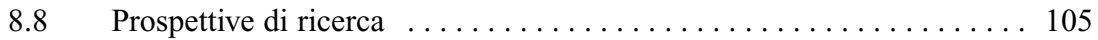

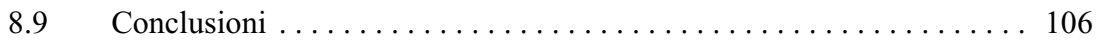

Bibliografia ................................ 107

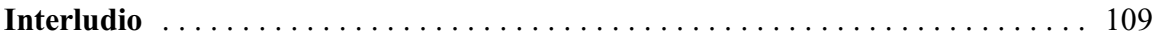

Fede e ragione di fronte al dolore innocente:

conversazione con Vito Mancuso $\ldots \ldots \ldots \ldots \ldots \ldots \ldots \ldots$

Enrico Molinari

Parte III Le esperienze cliniche e di ricerca $\ldots \ldots \ldots \ldots \ldots \ldots \ldots \ldots \ldots \ldots \ldots$

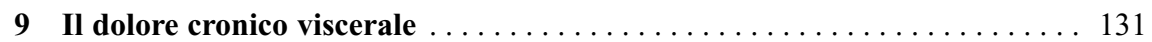

Mauro Ercolani, Alessandro Agostini

9.1 Caratteristiche del dolore viscerale $\ldots \ldots \ldots \ldots \ldots \ldots \ldots \ldots \ldots 1$

9.2 Dolore riferito e iperalgesia $\ldots \ldots \ldots \ldots \ldots \ldots \ldots \ldots \ldots \ldots \ldots \ldots$

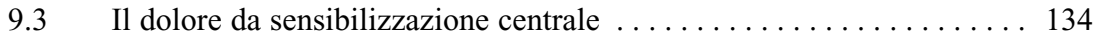

9.4 La sindrome dell'intestino irritabile (SII) $\ldots \ldots \ldots \ldots \ldots \ldots \ldots \ldots$

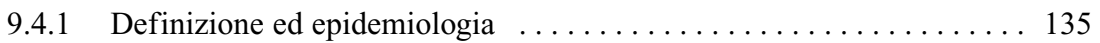

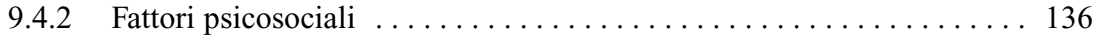

9.4 .3 Sintomi e segni . . . . . . . . . . . . . . . . . . . 136

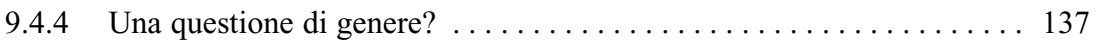

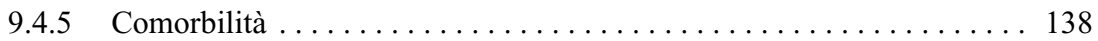

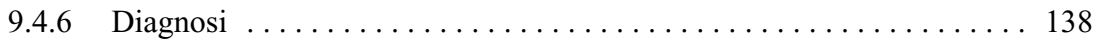

9.4 .7 Fattori genetici e ambientali . . . . . . . . . . . . . . . . . . 139

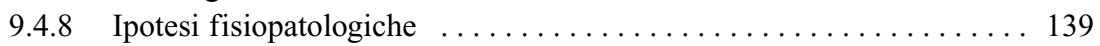

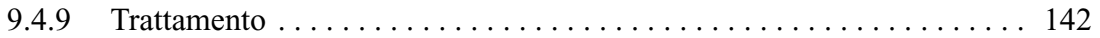

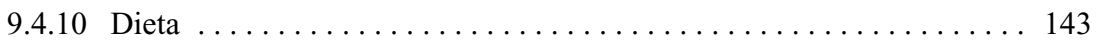

9.4 .11 Terapia farmacologica sintomatica $\ldots \ldots \ldots \ldots \ldots \ldots \ldots \ldots \ldots . \ldots \ldots$

9.4 .12 Terapie non farmacologiche . . . . . . . . . . . . . . . 145

9.4.13 Caratteristiche del professionista della psicoterapia $\ldots . \ldots \ldots \ldots . . .146$

Bibliografia ................................ 147

10 Clinica, metodologia e ricerca psicologica: applicazioni in un ambulatorio

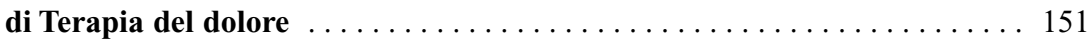

Andrea Cottini, Michele Monticelli, Carlo Nobili

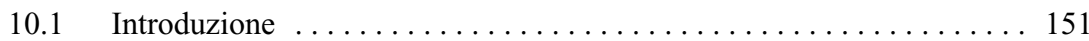

10.2 Contesto istituzionale e descrizione dell'attività ambulatoriale . . . . . 152

10.3 Modello medico-psicologico integrato . . . . . . . . . . . 153 


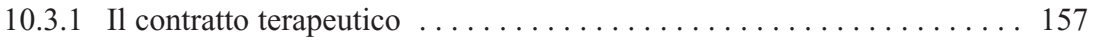

10.4 Rilassamento muscolare progressivo . . . . . . . . . . . . . . . . . 157

10.5 Psicoterapia breve . . . . . . . . . . . . . . . . . . . . . . . . . 158

10.6 Il progetto VIRNOPA: la realtà virtuale e l'ipnosi nel trattamento

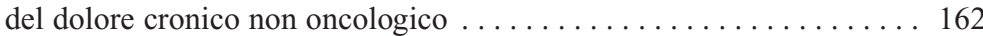

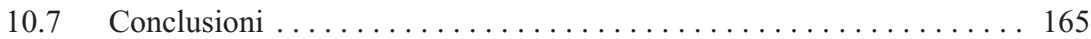

Ringraziamenti .............................. 165

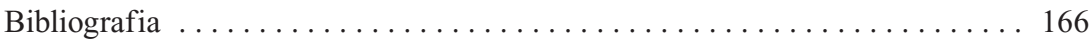

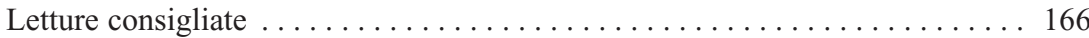

11 Servizio interno di Psicologia Clinica in un contesto ospedaliero ortopedico

Andrea Cottini, Daniela Ambrosi

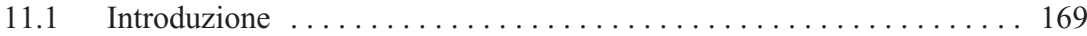

11.2 Servizio interno di Psicologia Clinica . . . . . . . . . . . . . . . . . . . 169

11.3 Unità Operativa di Medicina Riabilitativa $\ldots \ldots \ldots \ldots \ldots \ldots \ldots . \ldots 170$

11.4 Unità Operativa di Reumatologia . . . . . . . . . . . . . . . . . . . 172

11.4.1 Analisi di contesto . . . . . . . . . . . . . . . . . . . . . 173

11.4.2 Inquadramento psicologico e relazionale $\ldots \ldots \ldots \ldots \ldots \ldots \ldots \ldots$

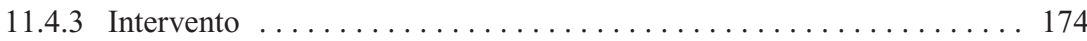

11.5 Conclusioni ...................................... 174

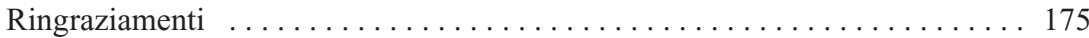

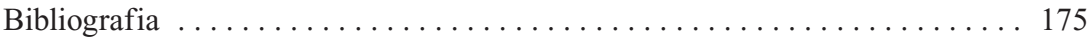

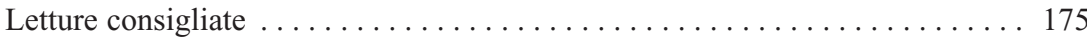

12 Dolore e obesità: una ricerca preliminare del Servizio di Psicologia Clinica dell'IRCCS Istituto Auxologico Italiano . . . . . . . . . . . . . . . . 177

Paola Cuzziol, Andrea Brioschi, Alessandro Mauro, Enrico Molinari,

Gianluca Castelnuovo

$12.1 \quad$ Premessa . . . . . . . . . . . . . . . . . . . . . . 177

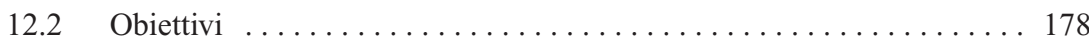

$12.3 \quad$ Materiali e metodi . . . . . . . . . . . . . . . . . . 178

12.3.1 Partecipanti ................................ 178

12.3.2 Strumenti . . . . . . . . . . . . . . . . . . . . . . . . . . . 179

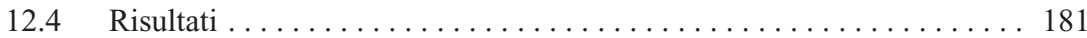

12.4.1 Analisi statistica . . . . . . . . . . . . . . . . . . . . . 181

12.4.2 Risultati . . . . . . . . . . . . . . . . . . . . . . . . . . 182

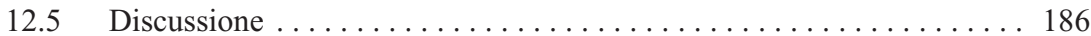

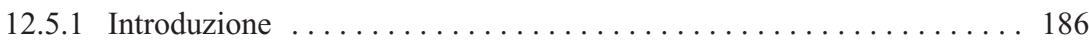

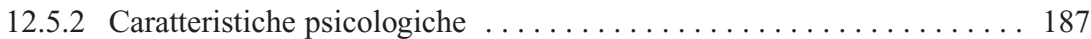

12.5.3 Valutazione psicofisiologica (MEDOC TSA-2001) . . . . . . . . . 189

12.5.4 Correlazioni fra dimensioni psicologiche e dati psicofisiologici . . . . . 190

12.6 Conclusioni . . . . . . . . . . . . . . . . . . . . . . . . . . . . . . . 191

Bibliografia .................................... 192 
13 L'esperienza del dolore nell'ambulatorio del medico di famiglia

Emanuele Zacchetti

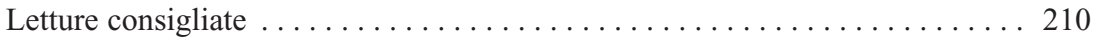

14 Le ultime frontiere per il trattamento del dolore: Realtà Virtuale 4D e lettino vibroacustico del Centro CARMA di Udine

Alberto Raffaelli, Diego Kriscak

$14.1 \quad$ Premessa . . . . . . . . . . . . . . . . . . . . . . . . . . . . 211

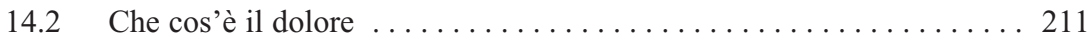

14.3 Realtà Virtuale come terapia . . . . . . . . . . . . . . . . . . . . 214

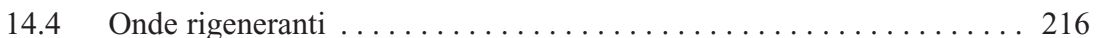

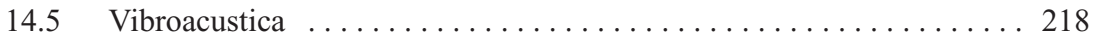

14.6 Strumentazione e percorso terapeutico . . . . . . . . . . . . . . . 223

14.6.1 Pazienti . . . . . . . . . . . . . . . . . . . . . . . . . . . . . . . . . . . 224

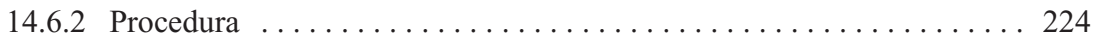

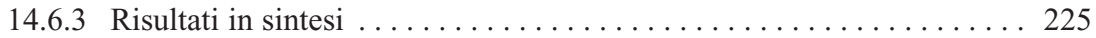

Bibliografia ................................. 226

15 Dolore cronico, dolore inutile. Prima indagine italiana della Onlus Nopain

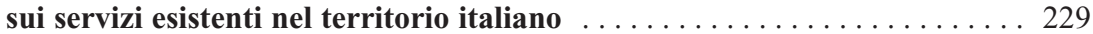

Paolo Notaro, Alessandra Voltolini, Adriano Meroni,

Maria Cesarina Montagna, Fabio Rubino

15.1 Introduzione . . . . . . . . . . . . . . . . . . . . . . . . . . . . . . . . 229

15.2 Definizione e dimensione del problema ............... 231

15.3 Raccomandazioni e modelli organizzativi internazionali . . . . . . . . . 234

15.4 Metodologia dell'indagine descrittiva . . . . . . . . . . . . . . . 236

$15.5 \quad$ Risultati . . . . . . . . . . . . . . . . . . . . . . . . . . . . . . . . . . . . 237

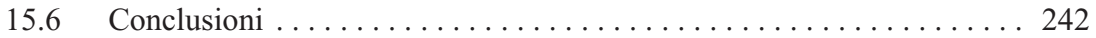

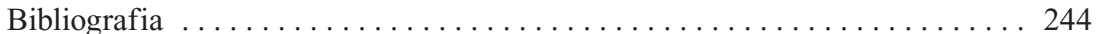

Letture consigliate . . . . . . . . . . . . . . . . . . . . . . . 245

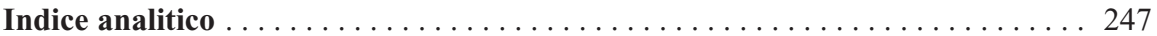




\section{Elenco degli Autori}

\section{Alessandro Agostini}

Dipartimento di Psicologia

Università di Bologna

Bologna

\section{Daniela Ambrosi}

Ambulatorio di Terapia del dolore

IRCCS Istituto Ortopedico Galeazzi

Milano

\section{Lara Bellardita}

Laboratorio di Psicologia

Università Cattolica del Sacro Cuore

Milano

\section{Andrea Brioschi}

Laboratorio di Ricerche di Neurobiologia IRCCS Istituto Auxologico Italiano

Ospedale S. Giuseppe

Piancavallo (VB)

\section{Gianluca Castelnuovo}

Laboratorio di Ricerche Psicologiche IRCCS Istituto Auxologico Italiano

Facoltà di Psicologia

Università Cattolica del Sacro Cuore

Milano

\section{Andrea Cottini}

Ambulatorio di Terapia del dolore

IRCCS Istituto Ortopedico Galeazzi

Milano

\section{Stefano Cugno}

Psicologo clinico

Milano

\section{Paola Cuzziol}

Laboratorio di Ricerche Psicologiche IRCCS Istituto Auxologico Italiano

Ospedale S. Giuseppe

Piancavallo (VB)

\section{Mauro Ercolani}

Dipartimento di Psicologia Clinica

Facoltà di Medicina e Chirurgia

Università di Bologna

Bologna

\section{Valentina Forni}

Psicologo clinico

Borghetto Lodigiano (LO)

\section{Diego Kriscak}

Centro CARMA srl

Tavagnacco (UD) 


\section{Vito Mancuso}

Facoltà di Filosofia

Università Vita-Salute San Raffaele

Milano

Alessandro Mauro

Dipartimento di Neuroscienze

Università di Torino

Laboratorio di Neurologia e

Neuroriabilitazione

IRCCS Istituto Auxologico Italiano

Adriano Meroni

Associazione Italiana per la cura

della malattia dolore

Onlus NOPAIN

Milano

\section{Enrico Molinari}

Laboratorio di Ricerche Psicologiche IRCCS Istituto Auxologico Italiano Facoltà di Psicologia

Università Cattolica del Sacro Cuore

Milano

\section{Maria Cesarina Montagna}

Associazione Italiana per la cura

della malattia dolore

Onlus NOPAIN

Milano

\section{Michele Monticelli}

Ambulatorio di Terapia del dolore

IRCCS Istituto Ortopedico Galeazzi

Milano

\section{Vincenzo Montrone}

U.O. di Fisiopatologia, Terapia del dolore e Cure Palliative

A.O. "Antonio Cardarelli"

Napoli

\section{Carlo Nobili}

Ambulatorio di Terapia del dolore

IRCCS Istituto Ortopedico Galeazzi

Milano

\section{Paolo Notaro}

S.S. di Terapia del dolore

A.O. Ospedale Niguarda Ca' Granda Milano

\author{
Alberto Raffaelli \\ Centro CARMA srl \\ Tavagnacco (UD) \\ Daniele Rovaris \\ Cooperativa Sociale Calypso ONLUS \\ Milano
}

\section{Fabio Rubino}

Associazione Italiana per la cura della malattia dolore

Onlus NOPAIN

Milano

\section{Mario Tavola}

S.S di Rianimazione

U.O. Anestesia e Rianimazione

Ospedale Civile A. Manzoni

Lecco

\section{Alessandra Voltolini}

Associazione Italiana per la cura

della malattia dolore

Onlus NOPAIN

Milano

\section{Emanuele Zacchetti}

Neurofisiologia clinica e Psicoterapia ASL 11 Vercelli

Borgo Sesia

Vercelli 